University of South Florida

DIGITAL COMMONS

Digital Commons @ University of

@ UNIVERSITY OF SOUTH FLORIDA

South Florida

\title{
New TOPEX Sea State Bias Models and Their Effect on Global Mean Sea Level
}

D. P. Chambers

University of Texas at Austin, donc@usf.edu

S. A. Hayes

University of Texas at Austin

J. C. Ries

University of Texas at Austin

T. J. Urban

University of Texas at Austin

Follow this and additional works at: https://digitalcommons.usf.edu/msc_facpub

Part of the Life Sciences Commons

\section{Scholar Commons Citation}

Chambers, D. P.; Hayes, S. A.; Ries, J. C.; and Urban, T. J., "New TOPEX Sea State Bias Models and Their Effect on Global Mean Sea Level" (2003). Marine Science Faculty Publications. 1379.

https://digitalcommons.usf.edu/msc_facpub/1379

This Article is brought to you for free and open access by the College of Marine Science at Digital Commons @ University of South Florida. It has been accepted for inclusion in Marine Science Faculty Publications by an authorized administrator of Digital Commons @ University of South Florida. For more information, please contact digitalcommons@usf.edu. 


\title{
New TOPEX sea state bias models and their effect on global mean sea level
}

\author{
D. P. Chambers, S. A. Hayes, J. C. Ries, and T. J. Urban \\ Center for Space Research, University of Texas at Austin, Austin, Texas, USA \\ Received 27 February 2003; revised 20 May 2003; accepted 11 July 2003; published 1 October 2003.
}

[1] During the calibration of the Jason-1 altimeter, it was discovered that the JasonTOPEX sea surface height (SSH) residuals contained a trend correlated with significant wave height (SWH), indicating an error in the sea state bias (SSB) model for one or both of the altimeters. After updating the SSB model for Jason the trend remained, which pointed to an error in the TOPEX model. Since two different TOPEX altimeters (TOPEX_A and TOPEX_B) have operated during the mission, we estimated new SSB models using data from each one. The estimated SSB model for TOPEX_B is significantly different than the model provided with the data, which was estimated using only TOPEX_A data. Replacing the SSB model on TOPEX with the new TOPEX_A and TOPEX_B models not only removes the correlation with SWH in the Jason-TOPEX SSH residuals, it also removes most of a bias between TOPEX_A and TOPEX_B that has been observed in tide gauge calibrations. The magnitude of the change between TOPEX_A SSH and TOPEX B SSH is of the order of $10 \mathrm{~mm}$ globally, with TOPEX B SSH increasing when the new SSB model is applied. The application of this improved model will increase the rate of observed global mean sea level rise from $1.7 \mathrm{~mm} / \mathrm{year}$ when the original TOPEX data are used to $2.8 \mathrm{~mm} /$ year when the updated SSB models are applied. INDEX TERMS: 4556 Oceanography: Physical: Sea level variations; 4215 Oceanography: General: Climate and interannual variability (3309); 4275 Oceanography: General: Remote sensing and electromagnetic processes (0689); 4594 Oceanography: Physical: Instruments and techniques; KEYWORDS: TOPEX, Jason-1 sea state bias, global mean sea level, altimeter calibration

Citation: Chambers, D. P., S. A. Hayes, J. C. Ries, and T. J. Urban, New TOPEX sea state bias models and their effect on global mean sea level, J. Geophys. Res., 108(C10), 3305, doi:10.1029/2003JC001839, 2003.

\section{Introduction}

[2] The TOPEX/Poseidon (T/P) radar altimeter mission was launched in August 1992. For over 10 years, it has been providing highly accurate measurements of sea surface height (SSH) and has contributed significantly to many topics of oceanography and geodesy $[F u$ and Cazenave, 2001]. Not counting the experimental Poseidon altimeter, two distinct TOPEX altimeters have operated during the mission. The TOPEX_A altimeter operated from shortly after launch to January 1999. After it was observed that there were large changes in the observed significant wave height (SWH) starting in mid-1997, a study by instrument engineers found a noticeable degradation in the TOPEX_A point-target-response (PTR) $[F u, 1998]$. It was recommended that TOPEX_A be turned off and the redundant backup (TOPEX_B) be turned on for at least a few cycles to see if this corrected the problem. This was done in February 1999. All tests indicated TOPEX_B performed as well as early TOPEX_A data, so the decision was made to keep TOPEX_B on as the primary science instrument for the

Copyright 2003 by the American Geophysical Union. 0148-0227/03/2003JC001839 remainder of the mission. The last $\mathrm{T} / \mathrm{P} 10$-day repeat cycle to use TOPEX_A was Cycle 235, ending on February 9, 1999. TOPEX_B was turned on at the beginning of Cycle 236 on the same day.

[3] Other than a few initial calibrations based on the first few months of data, no significant changes were made to the data algorithms, including the correction made to account for differences in the reflection of the radar pulse due to surface waves. This correction is usually referred to as the sea state bias (SSB), and is composed of several parts. One, the electromagnetic (EM) bias, is theoretically only a function of the frequency of the EM pulse. Since TOPEX_A and TOPEX_B have the same pulse frequencies (on both the primary $\mathrm{Ku}$-band and the secondary C-band), there should be no difference in the EM bias. The skewness and tracker biases are due to inaccurate tracking of the waveform by the on-board tracker, and also show dependence to the sea state and altimeter frequency [e.g., Chelton et al., 2001]. Since all three components are related to the sea state and altimeter frequency, in practice the combined SSB effect is estimated empirically by fitting data to a relationship between SSB, SWH, and wind speed (U) [e.g., Gaspar et al., 1994].

[4] The SSB model used for the TOPEX Merged Geophysical Data Records (MGDRs) was estimated by fitting 
TOPEX_A SSH crossovers for Cycles 1-110 to an empirical model of the form [Gaspar et al., 1994],

$$
\operatorname{SSB}=\operatorname{SWH}\left(\mathrm{a}_{0}+\mathrm{a}_{1} \mathrm{SWH}+\mathrm{a}_{2} \mathrm{U}+\mathrm{a}_{3} \mathrm{U}^{2}\right),
$$

and estimating the coefficients $a_{0}, a_{1}, a_{2}$, and $a_{3}$, where $\mathrm{SWH}$ is in $\mathrm{m}$ and $\mathrm{U}$ is in $\mathrm{m} / \mathrm{sec}$. At the time, this model best reduced the overall variance of the TOPEX crossover differences, which is interpreted as meaning it removes the majority of the SSB signal.

[5] The SSB model has an additional secondary effect on the SSH measurement. The radar signal is delayed by electrons in the ionosphere; the delay is a function of the radar frequency. Because of this, ranges are measured at two frequencies (one in the Ku-band, one in the $\mathrm{C}$-band) by both TOPEX and Jason-1. The electron content is the same for both paths, but the delay on the range is different. By combining the measured ranges after correcting for sea state effects [e.g., Chelton et al., 2001], an ionosphere correction is computed, which is then applied to the Ku-range measurement to correct for the path delay and obtain SSH. This means that the ionosphere correction is in part based on a Ku-band specific SSB model and a C-band specific SSB model. Thus any errors in either SSB model will cause an error in the ionosphere correction that will likely be correlated to the sea state. However, the contribution of SSB to the ionosphere is not $100 \%$, but is scaled by 0.175 for TOPEX and Jason-1 (based on a function of the Ku- and C-band frequencies). Thus an error in SSB of $1 \mathrm{~cm}$ will be a $2-\mathrm{mm}$ error in the ionosphere correction. Moreover, an empirical estimate of a SSB model (such as equation (1)) fit to SSH data containing such an error in the ionosphere correction will attempt to remove that portion of the signal that is correlated with SWH and wind speed, whether it is in $\mathrm{SSH}$, the ionosphere correction applied to $\mathrm{SSH}$, or both. Note also that while the SSH is computed from Ku-band ranges, the coefficients to equation (1) will represent the $\mathrm{Ku}$-band SSB and a portion of both the C-band and $\mathrm{Ku}$-band SSB error in the ionosphere correction. Thus, while the estimated model may not represent the "true" $\mathrm{SSB}$, it will attempt to minimize errors in the SSH (and ionosphere correction) related to the sea state.

[6] After TOPEX_B was turned on, the SSB model on the MGDRs was not changed, nor was there a change made in tracking or a skewness correction. This made an implicit assumption that not only were the EM biases the same between TOPEX_A and TOPEX_B, but that the skewness and tracker biases were also identical. Recent studies have focused on new types of SSB models estimated with TOPEX_A data [Gaspar and Florens, 1998; Gaspar et al., 2002] , theoretical EM bias models [Elfouhaily et al., 1999, 2000] or SSB models for the T/P follow-on mission, Jason-1. No work has been published on SSB models derived using only TOPEX_B data, although it was noted at the November $2000 \mathrm{~T} / \mathrm{P}$ Science Working Team Meeting by Gaspar et al. that a preliminary TOPEX_B SSB model differed significantly from the TOPEX_A model at very low SWH $(<2 \mathrm{~m})[F u, 2000]$.

[7] Jason-1 was launched on December 7, 2001. Over the next month, it was maneuvered into the same 10-day repeating ground track as $\mathrm{T} / \mathrm{P}$, but displaced in time by $70 \mathrm{~s}$. This was done in order to perform an unprecedented calibration mission with the TOPEX_B altimeter in order to ensure a continuity of highly accurate measurements and to enable the determination of any biases in the science system. Jason-1 began putting out Interim Geophysical Data Records (IGDRs) with Cycle 2 (starting on January 25, 2002), which corresponds to T/P Cycle 345.

[8] After describing the additional processing done to the standard TOPEX and Jason-1 IGDR data to make them as accurate and consistent as possible, we will discuss results from our analysis of the Jason-1 and TOPEX measurements. Contrary to expectations, the analysis pointed to evident problems in the TOPEX data and not just the Jason-1 observations. The errors were most significant in regards to global mean sea level (GMSL) studies.

\section{Data Processing}

[9] The TOPEX data are distributed in several formats. The IGDRs contain all information to compute SSH corrected for atmospheric, geophysical, and surface effects, but include an orbit that still has significant long-wavelength orbit error. The TOPEX IGDR data is available to the Science Working Team (SWT) within a few weeks of the end of a repeat cycle, but the MGDR (the most accurate data) is not produced for several months to a year later. However, MGDRs are essentially IGDRs except that (1) the orbit is replaced with a precise orbit ephemeris (POE), (2) the IGDR SSB model (a 1-parameter model) has been replaced with the model in equation (1) and coefficients estimated by Gaspar et al. [1994], and (3) a correction determined from the internal calibration mode has been applied to the range [Hayne et al., 1994]. The most difficult of these differences to obtain is the POE, but this is routinely computed at the University of Texas Center for Space Research (UTCSR) as part of the TOPEX verification [Tapley et al., 1994]. Therefore we routinely update the TOPEX IGDR data with the MGDR SSB model, the internal calibration correction, and the UTCSR POEs. Tests have shown that the difference between the MGDR-equivalent range and the MGDR range data agree within a few millimeters. We also add an additional correction that is not included in the MGDR. After initial MGDR production, it was discovered that one channel of the TOPEX Microwave Radiometer (TMR) was drifting, which caused a noticeable drift in SSH [Keihm et al., 2000]. We have applied an algorithm to correct for this drift based on global means (V. Zlotnicki, personal communication, 1999), which, in terms of SSH is

$$
\begin{aligned}
\mathrm{SSH}_{\mathrm{C}}= & \mathrm{SSH}_{\mathrm{M}}+(0.0012 \mathrm{~m} / \text { year })(\text { year }-1992.75), \text { year }<1997.0 \\
& \mathrm{SSH}_{\mathrm{C}}=\mathrm{SSH}_{\mathrm{M}}+0.0052 \mathrm{~m}, \text { year } \geq 1997.0
\end{aligned}
$$

where $\mathrm{SSH}_{\mathrm{M}}$ is the SSH from the TOPEX MGDRs (or IGDRs converted to MGDR quality) with all major corrections applied, and $\mathrm{SSH}_{\mathrm{C}}$ represents the $\mathrm{SSH}$ corrected for the effect of the TMR drift, which was initially estimated to level off after 1997. However, we should note that recent evaluations presented at SWT meetings suggest the TMR drift continued for some time after 1997, but that the updated corrections were not available for this study. The change is less than $1 \mathrm{~mm}$, so will not significantly affect the results of this study. 
[10] The Jason IGDRs contain all the data equivalent to the TOPEX IGDRs, except the initial orbits are significantly better. However, they still must be replaced by POEs in order to match the accuracy of the TOPEX MGDRs. Therefore we replace the orbits on the Jason-1 IGDRs with POEs computed at UTCSR.

[11] The Jason microwave radiometer (JMR), which measures path delay due to water vapor, was expected to need tuning relative to the TMR. However, the tuning has turned out to be relatively small, as initial statistics from the calibration mission indicate the untuned JMR and TMR agree at about $5 \mathrm{~mm}$ RMS of path delay, which is within the desired range. Therefore no additional corrections to the Jason-1 IGDR wet path delay have been made for this study. The preliminary Jason-1 SSB model was based on a new nonparameteric SSB model [Gaspar and Florens, 1998] fit to TOPEX, not Jason-1 data, and was an important correction to verify on orbit with real Jason-1 data.

[12] In order to compare the Jason-1 and TOPEX data during the calibration mission and to obtain the most accurate statistics, one needs to make one additional correction to the SSH. Although the two satellites are on the same nominal ground track spaced $70 \mathrm{~s}$ apart, they will not sample exactly the same location for several reasons. The ground tracks are continuously moving because of drag and other perturbing forces, and maneuvers are used to keep the true ground track within $\pm 1 \mathrm{~km}$ of the nominal track. Additionally, the sampling rates and orbital period of $\mathrm{T} / \mathrm{P}$ and Jason-1 differ slightly. Combined, all of these effects can cause the nearest sub-satellite points to differ by as much as 3-4 km. Because the geoid (and hence the mean sea surface, MSS) can have changes of several meters at these scales in some regions, the Jason- 1 and TOPEX data must be interpolated to a common point using a MSS model, or else one may measure the geoid difference between the two sub-satellite points and not the true difference measured in the SSH.

[13] We have collocated the TOPEX and Jason-1 SSH measurements to the same reference ground track using an along-track MSS model [Chambers et al., 1998a]. This collocates the measurements made at different times to the same spatial location, and reduces the short-wavelength geoid gradient signals. Any differences between the collocated Jason-1 and TOPEX SSHs (averaged globally over all available data) would therefore be attributable to biases or noise in the ranging system or corrections. To compute the relative bias, Jason-1 relative to TOPEX, we computed a $\mathrm{SSH}$ residual, $\Delta$, at each collocated point $(\mathrm{x}, \mathrm{y})$ and cycle $(\mathrm{t})$,

$$
\Delta(\mathrm{x}, \mathrm{y}, \mathrm{t})=\operatorname{SSH}_{\mathrm{J}}(\mathrm{x}, \mathrm{y}, \mathrm{t})-\operatorname{SSH}_{\mathrm{T}}(\mathrm{x}, \mathrm{y}, \mathrm{t}),
$$

where the SSH has been corrected for all recommended instrument, atmospheric, geophysical, and surface corrections except the inverted barometer (IB) correction and the ocean tide correction. The IB and tide corrections are real, instrument independent corrections, and should be nearly identical since the physical forces change much more slowly than $70 \mathrm{~s}$. Furthermore, the TOPEX and Jason-1 IGDRs use models for these corrections, which differ substantially. It was found that the standard deviation of the residuals (equation (3)) were significantly better when the

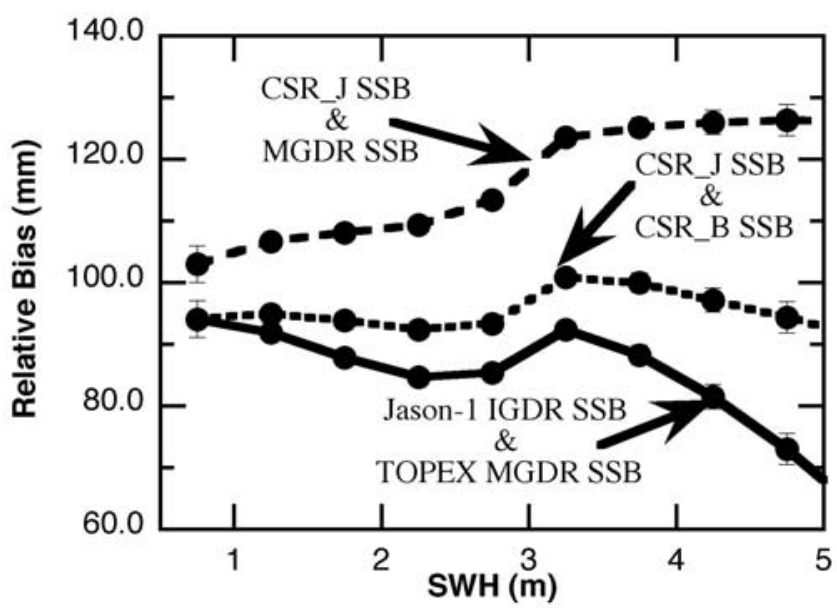

Figure 1. Estimated bias of Jason-1 SSH relative to TOPEX B SSH as a function of SWH. The labels indicate which SSB model was used to correct the SSHs. The error bars are the $99 \%$ confidence interval based on the formal error.

IB and tide models were not used than when they were, suggesting it was better to not include them.

\section{Results and Discussion}

[14] The SSH residuals (equation (3)) for all along-track collocated points were averaged globally for each 10-day cycle. Initial results were calculated with original Jason-1 IGDR models and data from Cycles 3-21 (February 4 through August 11, 2002), excluding Cycle 13 (because half the Jason-1 passes are missing) and Cycle 18 (because TOPEX_B was turned off for 10 days to allow a Jason-1 calibration with the Poseidon altimeter). The mean bias (the globally averaged residual) was relatively stable over all cycles $($ mean $=84.3 \mathrm{~mm}$, standard deviation $=1.7 \mathrm{~mm})$. However, when the residuals were averaged into $0.5-\mathrm{m}$ bins of the SWH instead of time, the relative bias was not stable as a function of SWH (Figure 1), indicating problems in the SSB model.

[15] As noted previously, the Jason-1 SSB model was preliminary and it was expected to be revised. While awaiting an official update, we estimated a parametric model of the form used for TOPEX; i.e., equation (1). The IGDR SSB model was removed from the Jason SSHs in our database, crossovers for Cycles 3-21 (including Cycle 13 and 18) were formed, and the coefficients to equation (1) were estimated using linear least squares [Gaspar et al., 1994]. When computing crossovers, the tide and IB corrections must be applied; here we have applied the Jason-1 IGDR models. Crossover residuals were computed by fitting a cubic spline to the $11 \mathrm{SSH}$ points along each ascending and descending pass, and then differencing the ascending value from the descending value at the crossover point. Crossovers were only calculated over water with a depth of more than $200 \mathrm{~m}$ and if there were at least eight points used in the cubic spline calculation. Any crossover residual with an absolute value larger than $50 \mathrm{~cm}$ was discarded. The estimated coefficients are given in Table 1 . The SSB calculated from equation (1) and the Jason-1 
Table 1. Estimated Coefficients of New Jason and TOPEX SSB Models $^{\mathrm{a}}$

\begin{tabular}{lrrr}
\hline Coefficient & \multicolumn{1}{c}{ CSR_J } & \multicolumn{1}{c}{ CSR_A } & \multicolumn{1}{c}{ CSR_B } \\
\hline $\mathrm{a}_{0}\left(\Delta \mathrm{a}_{0}\right)$ & $-4.05 \mathrm{E}-2$ & $1.79 \mathrm{E}-3$ & $-3.17 \mathrm{E}-3$ \\
$\mathrm{a}_{1}\left(\Delta \mathrm{a}_{1}\right)$ & $2.57 \mathrm{E}-3$ & $3.64 \mathrm{E}-5$ & $2.51 \mathrm{E}-4$ \\
$\mathrm{a}_{2}\left(\Delta \mathrm{a}_{2}\right)$ & $-2.55 \mathrm{E}-3$ & $-4.56 \mathrm{E}-4$ & $1.53 \mathrm{E}-4$ \\
$\mathrm{a}_{3}\left(\Delta \mathrm{a}_{3}\right)$ & $-9.64 \mathrm{E}-4$ & $1.78 \mathrm{E}-5$ & $-2.44 \mathrm{E}-5$ \\
\hline
\end{tabular}

${ }^{a}$ The coefficients for CSR_J are total coefficients $\left(a_{0} \ldots a_{1}\right)$, while the coefficients for CSR_A and $\overline{C S R} \_B$ are delta coefficients $\left(\Delta \mathrm{a}_{0} \ldots \Delta \mathrm{a}_{3}\right)$ relative to the original MGDR model.

coefficients from Table 1 should be subtracted from the uncorrected Jason-1 SSH measurement in order to apply the updated SSB correction.

[16] The updated Jason-1 SSB model differs significantly with the one used for TOPEX particularly in the dependence on SWH. The TOPEX data indicate a $2-2.5 \%$ dependence on SWH, which is close to theoretical EM-bias for the Ku-band frequency, while Jason-1 data show a 4\% dependence. This discrepancy is still a matter of debate among the SWT and is under investigation. However, it has been pointed out (R. Schroo, personal communication, 2002) that among all the altimeters flown to data, only TOPEX has such a low percentage SWH dependence.

[17] When this new SSB model (hereinafter referred to as CSR_J SSB) was applied to the Jason-1 data, the alongtrack collocated residuals were re-computed and averaged as both a function of time and SWH. The globallyaveraged mean over all cycles changed significantly (84.3 to $116.3 \mathrm{~mm}$ ), but the variation from cycle to cycle was similar (standard deviation $=2.0 \mathrm{~mm}$ ). However, there was still an apparent relationship between the relative bias and SWH (Figure 1), indicating further SSB error in either Jason-1 or TOPEX.

[18] It is possible that error remains in the new Jason-1 SSB model, since only a relatively small sample of SWH and wind speed were sampled in the fit. However, various models fit to subsets of the Jason-1 crossover data all showed a similar trend. This led us to consider the possibility of residual SSB error in TOPEX.

[19] Recall that the TOPEX SSB model was estimated from TOPEX_A data, and then applied to both TOPEX_A and TOPEX_ $\overline{\mathrm{B}}$ data as if the instrument had not changed. If the SSB had not changed, then estimates from the same number of TOPEX_A cycles and TOPEX_B cycles using the same editing criteria and procedures should produce the same, or reasonably similar, coefficients.

[20] Therefore, for our new analysis of both TOPEX_A and TOPEX_B, we estimated correction terms to the original TOPEXX SSB model (equation (1)) as

$$
\Delta \mathrm{SSB}=\operatorname{SWH}\left[\Delta \mathrm{a}_{0}+\Delta \mathrm{a}_{1} \mathrm{SWH}+\Delta \mathrm{a}_{2} \mathrm{U}+\Delta \mathrm{a}_{3} \mathrm{U}^{2}\right],
$$

since equation (1) is linear in terms of the coefficients $a_{0}, a_{1}$, $\mathrm{a}_{2}$, and $\mathrm{a}_{3}$. We used crossovers for TOPEX Cycles 11-158 (TOPEX_A) and Cycles 240-349 (TOPEX_B). The same estimation procedure as with the Jason-1 crossovers was used. The TOPEX_A data all occur before the noted PTR degradation, while the TOPEX-B data started a few cycles after the new instrument was turned on to allow for any power-on associated drifts to have stopped.
[21] The delta coefficients $\left(\Delta \mathrm{a}_{0}, \Delta \mathrm{a}_{1}, \Delta \mathrm{a}_{2}, \Delta \mathrm{a}_{3}\right)$ for the new TOPEX models (hereinafter referred to as CSR_A and CSR_B) are shown in Table 1 . The $\triangle$ SSB calculated from equation (4) should be subtracted from the TOPEX SSH measurement corrected with the MGDR correction SSB model in order to apply the updated correction. There is evidence of a small bias in the TOPEX data that is range-rate dependent (P. Callahan, personal communication, 2003); that is, it changes if the satellite is moving away from or toward the equator. By estimating the SSB with consistent ascending-descending pass crossovers, this might alias the signal into the SSB. However, when we calculate crossovers by time (i.e., time1 - time2, time2 > time1), and re-estimate the SSB coefficients, the difference is at most in the last significant digit. This suggests the effect of any range-rate error on the SSB model is negligible.

[22] The changes in CSR_A from the MGDR model are significantly smaller than for CSR B, as was expected. The percent change in the coefficients for CSR A ranges from a low of $1 \%$ to a high of about $10 \%$, while the change for CSR B ranges from a low of $4 \%$ to more than $16 \%$. The change in the coefficient scaling SWH $\left(\mathrm{a}_{0}\right)$ in CSR_B is twice as large as that in CSR_A. For the combination of SWH and wind speed with the highest percentage of observations globally ( $\mathrm{SWH}=2 \mathrm{~m}, \mathrm{U}=7.25 \mathrm{~m} / \mathrm{s}$ ), the SSH bias change due to CSR_A is only $1 \mathrm{~mm}$, while the change is nearly $6 \mathrm{~mm}$ for CSR_B.

[23] If the CSR_B model is applied to the TOPEX data and the Jason-1 relative bias is recalculated, the result is another change in the mean bias to $95.0 \mathrm{~mm}$ (standard deviation $=1.8 \mathrm{~mm}$ ), but the relative bias as a function of SWH is now reasonably flat (Figure 1), indicating no significant residual error in the SSB models. The kink observed in all three lines at $3 \mathrm{~m} \mathrm{SWH}$ is an artifact of the tracker gate on the TOPEX data (P. Callahan, personal communication, 2002), and cannot be removed with a parametric SSB model as used here.

[24] While these results are highly suggestive that different SSB models should be used for TOPEX_A and TOPEX_B, another test further supports this conclusion. For several years, TOPEX data along passes near tide gauges have been compared to the tide gauge measurements to look for drifts or changes in the altimeter data [Chambers et al., 1998b; Mitchum, 1998, 2000]. Mitchum [2000], in particular, has devoted significant effort to develop a processing procedure in order to minimize the differences between the two measurement types and extract as much information as possible. We have modified our tide gauge calibration procedure to be more similar with the one Mitchum [2000] uses, with the exception that we use only a glacial isostatic adjustment (GIA) correction and no additional land motion correction, as well as a slightly different tide gauge network [Hayes, 2002; T. J. Urban et al., Multi-satellite global mean sea level using relative bias determinations from tide gauges, manuscript in preparation, 2003]. Although we have not used a complete land adjustment model, our results (Figure 2) show a distinct bias in the residuals after the switch to TOPEX_B from TOPEX_A when the MGDR SSB model is used, without large drifts on either side. The value of the jump ranges from 3 to $7 \mathrm{~mm}$ (TOPEX_B SSH lower than TOPEX_A) 


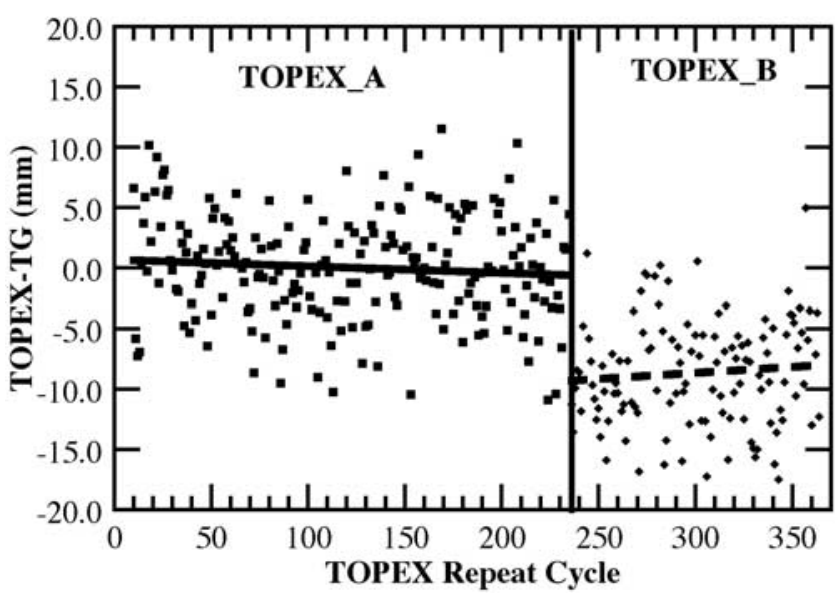

Figure 2. Cycle averages of TOPEX-tide gauge (TG) residuals where TOPEX SSH has been corrected with the MGDR SSB model. The solid and dashed lines are linear fits to the TOPEX_A and TOPEX_B residuals respectively.

depending on the calibration method and the technique used for determining the bias (mean matching, end-point matching, etc.). Although the fact that we have not applied a complete land motion adjustment may emphasize this jump somewhat, we note that although Mitchum [2000] incorporates a more robust land motion model, the results of that analysis have a very similar behavior at the TOPEX_A/TOPEX_B switch (G. Mitchum, personal communication, 200立).

[25] Note that a calibration of the TOPEX_B altimeter was conducted shortly after it was turned on and that corrections totaling approximately $100 \mathrm{~mm}$ were applied to bring the data mostly in line with the TOPEX A data (P. Callahan, personal communication, 2003). In light of this, a 3- to 7-mm residual offset is not unreasonable. A portion of the bias is also undoubtedly an effect of the SSB error on the ionosphere correction.

[26] When the new CSR A and CSR_B models are applied, the difference is reduced (Figure 3 ), so that the break between TOPEX_A and TOPEX_B is no longer readily apparent. Since only the SSB models were changed and nothing else, the bias can only be due to the SSB model (or the portion of the ionosphere error absorbed by the SSB model). Thus it appears that the apparent residual bias between TOPEX_A and TOPEX_B using the MGDR data is essentially removed with an updated sea state bias. This bias is most likely in the tracker bias portion, because the EM biases should be the same.

[27] The change in global mean sea level (GMSL) computed from the TOPEX data on the MGDR and that corrected for the new SSB models is even larger than the change at the tide gauge sites (Figure 4). The GMSL after February 1999 moves up by $7.3 \mathrm{~mm}$ after the new SSB models are used (standard deviation $=0.4 \mathrm{~mm}$ ), compared to a 5-mm change at the tide gauge sites. Part of this is due to a sampling difference in the average SWH and wind speed at the tide gauge sites compared to the global average. The linear rate of GMSL increases by more than $1 \mathrm{~mm} /$ year when the new SSB models are used, from $1.7 \mathrm{~mm} /$ year before to $2.8 \mathrm{~mm} /$ year after.

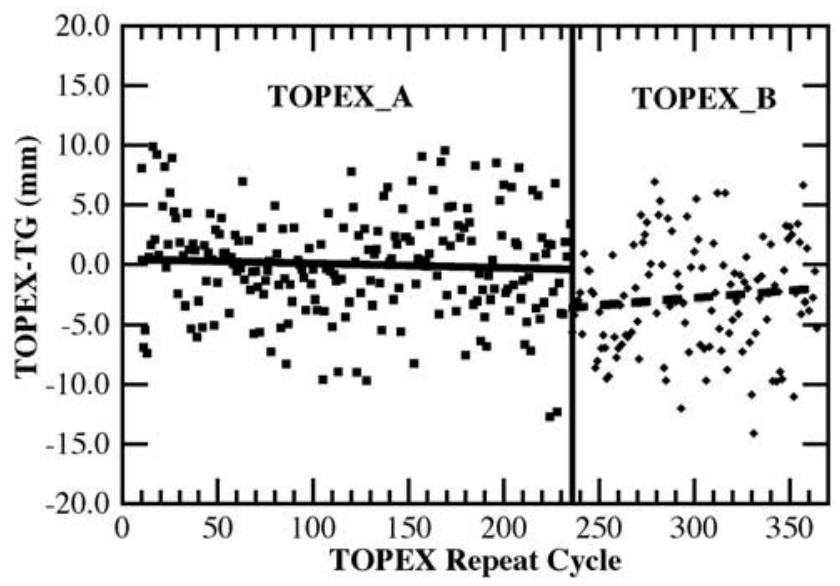

Figure 3. Same as Figure 2, except that the TOPEX SSHs have been corrected for CSR_A and CSR_B SSB models.

[28] Several researchers have used tide gauge calibration results to correct for this bias in TOPEX GMSL [e.g., Nerem and Mitchum, 2001], under the assumption that the bias is globally uniform. However, as this research suggests, the bias is not uniform globally, but is strongly dependent on the SWH and wind speed. Thus, correcting the TOPEX data with a new SSB model that is different for Side A and Side B is preferable to applying a single global correction.

[29] Although the SSB model presented here removes a large amount of the error in the SSH measurement after the switch to TOPEX_B, it will undoubtedly not remove all the error, due to its smooth nature. Another problem, as noted by Gaspar and Florens [1998], is that while SSB models of the form of equation (1) do minimize the variance of the crossovers, they may still be significantly different from the "true" solution, by up to several centimeters at high SWH. It is for this reason that Gaspar and Florens [1998] studied nonparameteric SSB models; it is probable that they will better find the "true" SSB. No consistent nonparameteric models for both TOPEX A and TOPEX B have been published, but some preliminary models have been made

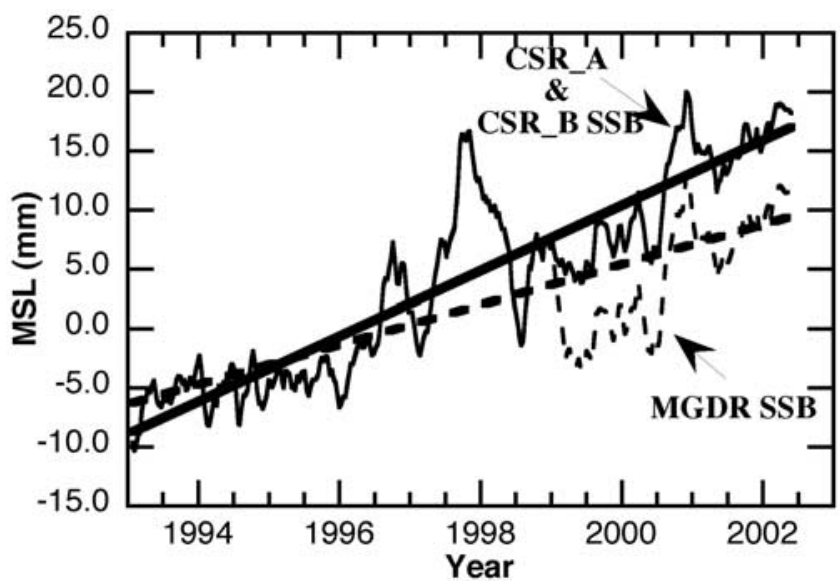

Figure 4. GMSL from TOPEX when corrected for MGDR SSB (dashed curve) and CSR_A and CSR_B SSB models (solid curve). The lines are best fit linear trends. 


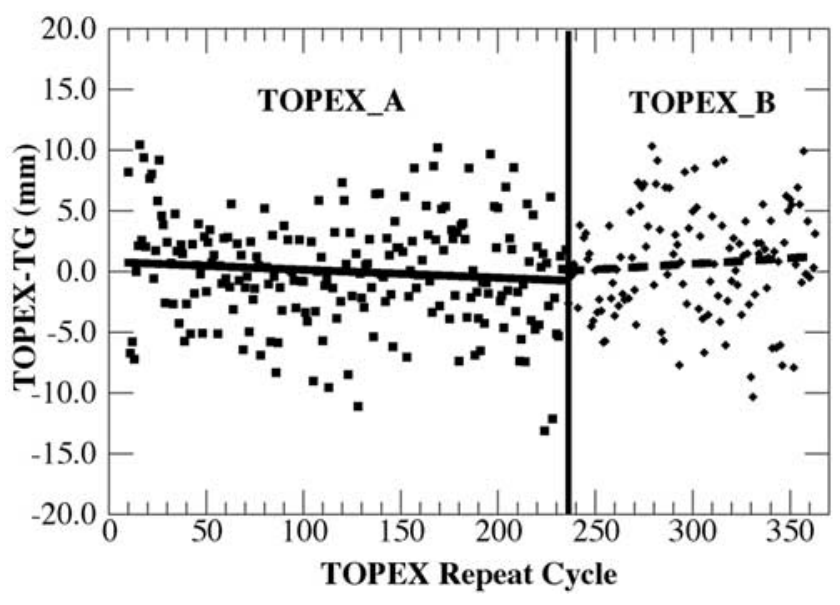

Figure 5. Same as Figure 2, except that the TOPEX SSHs have been corrected for TOPEX_A and TOPEX_B nonparameteric models provided by S. Labroue.

available for Jason-1 calibration efforts (S. Labroue, personal communication, 2002). The results are encouraging (Figure 5), and suggest that a nonparameteric model may be able to remove the bias between TOPEX_A and TOPEX_B more effectively than the model presented here, although the difference in GMSL is only a few millimeters. Perhaps more importantly, a nonparameteric SSB model should be able to better compensate for the error caused by the tracker at $3 \mathrm{~m} \mathrm{SWH}$ (and at other gates), thereby producing flatter curves than in Figure 1. It must be emphasized that the use of a nonparameteric SSB model for either TOPEX or Jason-1 will cause yet another change in the Jason-1 relative bias, and that the nonparameteric models are computed from data which have an ionosphere correction applied based on an older, incorrect SSB model [Gaspar and Florens, 1998].

[30] If the tracker bias is the problem, then retracking the raw waveforms [e.g., Rodriquez and Martin, 1994] is probably a preferable method to an empirical SSB model. If the data are retracked properly, then the SSB model should represent mostly the EM bias, and thus be the same for TOPEX_A and TOPEX_B. A preliminary set of retracking corrections to some of the TOPEX_B data during the overlap with Poseidon-1 have been made available (P. Callahan, personal communication, 2002). Analysis of these data indicates that the retracking process removes as much of the apparent TOPEX_B bias as the new SSB models do, but further tests need to be done to verify this.

\section{Conclusions}

[31] It has been demonstrated that much of the apparent bias in the TOPEX_B data is caused by using the same SSB model as for TOPEX_A. Computing a different SSB model for TOPEX_B will reduce this bias significantly. The error caused by not correcting for this bias properly can vary from 1 to $10 \mathrm{~mm}$ locally, depending on the wave height and wind speed, and about 7-8 $\mathrm{mm}$ globally. Correcting for the error with a global bias will remove the gross error, but may still be off by several millimeters locally. For investigations dealing with long-term sea level change, this can be a significant error.
[32] New SSB models to correct for the error have been presented (Table 1). A preliminary SSB model for the Jason-1 data is also presented. When these models are used for the TOPEX and Jason-1 data, the estimated Jason-1 relative bias is essentially uniform in time and as a function of SWH and wind speed. Although the SSB models presented here are of a similar form as that used for the TOPEX MGDR data, they probably will not model the true SSB. Part of this is because of their smooth, parametric form, while part of it is due to the fact that they have absorbed sea state error introduced through the ionosphere correction. Investigations with a newer less smooth nonparametric SSB model suggest it may be slightly better at removing the TOPEX B bias. However, this type of model will still be affected by the SSB error in the ionosphere correction. Since the Ku-band SSB for TOPEX_B and Jason-1 appear to be significantly different than the models provided, the C-band models are also likely incorrect. Ideally, this procedure should be iterated, where a SSB model is estimated for both $\mathrm{Ku}-$ and $\mathrm{C}$-band data, a new ionosphere correction is computed and applied, then the SSB models updated, etc., until the results do not change significantly. This should be investigated in future studies. However, for most users, the model presented here is easy to implement immediately and will correct for the majority of the problems related to the sea state.

[33] It is also very important to point out another issue regarding changing SSB models: that is the biasing of various SSH reference models. It has already been demonstrated how large an effect a change in SSB model has on the relative bias estimation between Jason-1 and TOPEX. Values change by several centimeters. There is a similar bias introduced to reference MSS models. Most global MSS models have used TOPEX A and TOPEX B data corrected for the old MGDR SSB. Since the SSB error has the effect of a local bias, a portion of the TOPEX_B SSB error has likely aliased into the MSS model. Most of this is likely in the mean height, and can be corrected for by re-estimating the local mean after changing the SSB model. However, some of the SSB error may be aliased into the MSS gradients. Further study is needed to quantify this error.

[34] We should also point out that for many studies of sea level variations using TOPEX data, the error introduced by not using a TOPEX_B specific SSB model may be significantly smaller than the signal of interest. For the average range of SWHs $(1-3 \mathrm{~m})$ and wind speeds $(5-10 \mathrm{~m} / \mathrm{s})$, the height error will be approximately $3-10 \mathrm{~mm}$. The error will also mostly be a local bias between the first part of time series and the latter part (jumping at February 1999). Any research based on data solely before February 1999 will not be affected by the problem discussed here. Also, anyone who removes trends from the data will also tend to minimize the problem. The new models produced here are most significant for research into GMSL change, where $0.5 \mathrm{~mm} /$ year changes are important, and for MSS modeling, where stable (constant biased) references are preferred.

[35] Acknowledgments. The authors would like to thank B. Beckley, P. Callahan, S. Desai, P. Gaspar, S. Labroue, E. Leuliette, S. Nerem, P. Vincent, and V. Zlotnicki for countless discussions on the TOPEX and Jason-1 SSB models. The free exchange of different models, comments, and ideas was extremely useful. This research was supported in part by 
NASA grants NAG5-9144, NAG5-11636, NASA JPL grant 961429, and NOAA grant 50-SPNA-1-00.

\section{References}

Chambers, D. P., B. D. Tapley, and R. H. Stewart, Reduction of geoid gradient error in ocean variability from satellite altimetry, Mar. Geod. 21, 25-39, 1998a.

Chambers, D. P., J. C. Ries, C. K. Shum, and B. D. Tapley, On the use of tide gauges to calibrate altimeter drift, J. Geophys. Res., 103, 12,885$12,890,1998 \mathrm{~b}$.

Chelton, D. B., J. C. Ries, B. J. Haines, L. L. Fu, and P. S. Callahan, Satellite altimetry, in Satellite Altimetry and Earth Science, Int. Geophys. Ser., vol. 69, edited by L. L. Fu and A. Cazanave, pp. 1-131, Academic, San Diego, Calif., 2001.

Elfouhaily, T., D. R. Thompson, D. Vandemark, and B. Chapron, Weakly nonlinear theory and sea state bias estimations, J. Geophys. Res., 104, 7641-7647, 1999.

Elfouhaily, T., D. R. Thompson, B. Chapron, and D. Vandemark, Improved electromagnetic bias theory, J. Geophys. Res., 105, 1299-1310, 2000.

Fu, L. L., (Ed.), Minutes of the Joint TOPEX/POSEIDON Jason-1 Science Working Team Meeting, JPL Rep. D-16608, pp. 8-9, Jet Propul. Lab., Pasadena, Calif., 1998.

Fu, L. L., (Ed.), Minutes of the Third Joint TOPEX/POSEIDON and Jason1 Science Working Team Meeting, JPL Rep. D-20240, pp. 32-33, Jet Propul. Lab., Pasadena, Calif., 2000.

Fu, L. L., and A. Cazenave (Eds.), Satellite Altimetry and Earth Science, Int. Geophys. Ser., vol. 69, Academic, San Diego, Calif., 2001.

Gaspar, P., and J. P. Florens, Estimation of the sea state bias in radar altimeter measurements of sea level: Results from a new nonparametric method, J. Geophys. Res., 103, 15,803-15,814, 1998.

Gaspar, P., F. Ogor, P.-Y. Le Traon, and O. Z. Zanife, Estimating the sea state bias of the TOPEX and POSEIDON altimeters from crossover differences, J. Geophys. Res., 99, 24,981-24,994, 1994.
Gaspar, P., S. Labroue, F. Ogor, G. Lafitte, L. Marchal, and M. Rafanel, Improving nonparameteric estimates of the sea state bias in radar altimeter measurements of sea level, J. Atmos. Oceanic Technol., 19, 16901707, 2002.

Hayes, S. A., The determination of relative biases between satellite altimeters using tide gauge data, Tech. Memo. CSR-TM-02-03, Cent. for Space Res., Austin, Tex., 2002.

Hayne, G. S., D. W. Hancock, and C. L. Purdy, TOPEX altimeter range stability estimated from calibration mode data, in TOPEX/POSEIDON Research News, JPL 410-42(3), pp. 18-22, Jet Propulsion Lab., Pasadena, Calif., 1994.

Keihm, S. J., V. Zlotnicki, and C. S. Ruf, TOPEX microwave radiometer performance evaluation, IEEE Trans. Geo. Remote Sens., 38, 13791386,2000 .

Mitchum, G. T., Monitoring the stability of satellite altimeters with tide gauges, J. Atmos. Oceanic Technol., 15, 721-730, 1998.

Mitchum, G. T., An improved calibration of satellite altimetric heights using tide gauge sea levels with adjustment for land motion, Mar. Geod., $23,145-166,2000$.

Nerem, R. S., and G. T. Mitchum, Observations of sea level change from satellite altimetry, in Sea Level Rise: History and Consequence, edited by B. C. Douglas, M. Kearney, and S. Leatherman, pp. 121 -164, Academic, San Diego, 2001.

Rodriquez, E., and D. W. Martin, Assessment of TOPEX algorithm performance using waveform retracking, J. Geophys. Res., 99, 24,957-24,969, 1994.

Tapley, B. D., et al., Precision orbit determination for TOPEX/POSEIDON, J. Geophys. Res., 99, 24,383-24,404, 1994.

D. P. Chambers, S. A. Hayes, J. C. Ries, and T. J. Urban, Center for Space Research, University of Texas at Austin, 3925 West Braker Lane, Suite 200, Austin, TX 78759-5321, USA. (chambers@csr.utexas.edu; hayes@csr.utexas.edu; ries@csr.utexas.edu; urban@csr.utexas.edu) 